im Verlaufe anderer schwerer Krankheiten, wie Typhus, Variola, Masern, Erysipel, Dysenterie, sowie im Anschluss an Skorbut, Pemphigus, Herpes und bei dem jetzt ausgestorbenen Hospitalbrand ist sie beobachtet worden. Von dieser sekundären Form der Rachengangrän ist hier nicht die Rede. Es handelt sich allein um die primäreidiopathische Angina gangraenosa, einer Krankheit sui generis.

In der mir erreichbaren Litteratur ${ }^{1}$ ) habe ich nur 22 reine Fälle dieser Krankheit aufgefunden, die fast sämmtlich von ausländischen Autoren publizirt sind. Nur ein kursorisch mitgetheilter Fall wurde in Deutschland beobachtet. Die ausfïhrliche Kasuistik entstammt in erster Linie der Feder französischer Autoren, die zusammen zwölf Fälle beschrieben haben. Zwei ausführliche Beobachtungen stammen aus Russland, zwei aus Spanien und fünf aus England.

Die Geschichte der primären Angina gangraenosa reicht bis in die zweite Hälfte des 18. Jahrhunderts zurück. Damals und noch in den ersten zwei Dezennien des vorigen Jahrhunderts galt sie in Frankreich als durchaus nicht selten. Doch war, was zu jener Zeit primäre Rachengangrän hiess, wohl meistens Diphtherie. Erst Bretonneau machte 1826 in seiner berühmten Schrift über die Diphtherie dieser Verwechselung ein Ende. Er wies nach, dass der sogenannte Rachenbrand fast stets echte Diphtherie sei, dass es andererseits bei der Diphtherie zu sekundärer brandiger Zerstörung der Rachentheile kommen könne, dies aber ein äusserst seltenes Ereigniss sei.

Die durch ihn erzeugte Umkehr der Meinungen führte, wie oftmals in der Medizin, zur Uebertreibung nach der anderen Seite. Jetzt wollte man von primärer Angina gangraenosa durchaus nichts mehr wissen, und weitergehend als Bretonneau selbst, leugneten nunmehr die Aerzte ihr Vorkommen gänzlich. ${ }^{2}$ )

Es ist das Verdienst von Gubler (2) und Trousseau (4), sie von neuem beschrieben und ihre allgemeine Anerkennung und nosologische Selbständigkeit gesichert zu haben.

Die erste genaue Schilderung der Krankheit ist Trousseau (4) zul danken. Sie mag hier in gekürzter Form ihren Platz finden, unnsomehr als die neueren Autoren, die dieses Krankheitsbild entworfen haben, wie Mackenzie (7), Blumenau (12), Kronenberg (13), sich in den wesentlichen Punkten an Trousseau anlehnen.

Der Grundcharakter der primären brandigen Angina besteht nach Trousseau in einer Nekrose der Rachenschleimhaut, die manchmal auf Wangen und Lippen - ähnlich dem Wasserkrebs übergreifen kann. Sie ist unabhängig von früheren Krankheiten und von epidemischen Einflüssen. Sie befällt zuweilen scheinbar ganz Gesunde ohne erkennbare Ursache. Sie führt oft und verschieden schnell zum Tode. Anatomisch kennzeichnet sich nach Trousseau die Gangrän durch graue, schwärzliche oder ganz schwarze Plaques mit scharfen gelblichen Rändern, die erhaben erscheinen, wenn sich der Schorf losgelöst hat. Die vom Brande ergriffenen Weichtheile werden gänzlich zerstört und es bilden sich unter dem Brandschorfe mehr oder minder tiefe Ulzerationen. Der Brand kann sich auf eine circumskripte Stelle beschränken, geht aber zuweilen auf die angrenzenden Partieen, Gaumensegel, Zäpfchen, hintere Rachenwand, Ligg. ary-epiglottica über. Die Schleimhaut in der Umgebung der brandigen Theile hat ein livides, dunkelroth-violettes Aussehen und ist ödematös geschwollen.

Charakteristisch ist der starke Foetor ex ore, der zuweilen an Kothgeruch erinnert. Es besteht heftiger Schmerz, besonders beim Schlucken, kann aber auch, wie in dem von Trousseau selbst geschilderten Falle fehlen. Die Stimme ist näselnd, auch dann, wenn der weiche Gaumen nicht befallen ist. Die Halsdrüsen schwellen an. Im Gegensatz zur Diphtherie kann diese Schwellung aber auch fehlen.

Klinisch äussert sich die Krankheit durch die allerschwersten

\section{Primäre Angina gangraenosa bei einem Knaben.}

Von Dr. E. Oberwarth in Berlin.

Im Nachstehenden wird die Krankheitsgeschichte eines zwölfjährigen Knaben berichtet, welcher an primärer Angina gangraenosa maligna gelitten hat und nach vielwöchigem Kampfe dieser seltenen und tückischen Krankheit zum Opfer gefallen ist.

Es ist bekannt, dass Gangrän des Pharynx als gefürchtete Complikation der Diphtherie und des Scharlach vorkommt. Auch Allgemeinerscheinungen, welche die Bösartigkeit des Erregers und

J) s. Zusammenstellung am Schlusse.

2) 1841 veröffentlichten Rilliet und Barthez (Archiv. génér. de Méd., 3. série, tome 12, p. 446) einige sehr interessante Beobachtungen von Gangrän des Rachens bei Kindern, die aber nicht hierher gehören, da es sich um sekundären, im Anschluss an Allgemeinerkrankungen aufgetretenen Rachenbrand handelte. Noch 1853 sagen dieselben Autoren: (Traité des maladies des enfants, 2. Auflage, Bd.II, p. 398) „Nach unseren Beobachtungen ist die Rachengangrän immer eine sekundäre Affektion." 
die Vergiftung des ganzen Organismus bekunden. Verdauung und Appetit leiden schwer. Fieber fehlt, vielmehr sinkt die Körperwärme bedeutend, das Hautkolorit wird bläulich. Der Herzschlag ist stark verlangsamt. Der Tod erfolgt im Collaps bei bis zum Schluss erhaltenen Bewusstsein oder im Coma nach mehrtägiger Benommenheit des Sensoriums. - Soweit Troussea u. Seine Schilderung ist nach den übrigen Autoren folgendermaassen zu vervollständigen:

Die Aetiologie ist bislang unklar. Epidemische Einflüsse scheinen ausgeschlossen. Lastra (10) hat in seinen beiden Fällen massenhaft Leptothrix buccalis im Mundschleim und Mandelbelag gefunden und stellt die Frage der ätiologischen Bedeutung dieses Pilzes zur Diskussion. Sonstige positive bakteriologische Befunde sind nicht vorhanden, das Fehlen der Löffler'schen Diphtheriebazillen wird betont.

Die Disposition scheint bei Erwachsenen grösser als bei Kindern zu sein. Von ersteren war das männliche Geschlecht stärker betroffen. 13 Fälle mit bezüglichen Angaben vertheilen sich auf zehn Männer und drei Frauen. Bei den Kindern besteht das umgekehrte Verhältniss; es waren von vier Kindern drei Mädchen, bei dem vierten fehlt die Angabe des Geschlechts. Das Alter schwankt bei den Erwachsenen von 19-38 Jahren, von den Kindern waren drei unter vier, eins 12 Jahre alt. Die Mortalität betrug $50 \%$. Von den 22 Fällen der Litteratur starben 11.

Ueber Prodrome wird nichts Charakteristisches vermerkt. Halsschmerzen, Röthung und Schwellung der Rachenorgane, Stechen in einer Mandel, Rachen- und Kehlkopfkatarrhe sind zuweilen wochen- oder monatelange Vorläufer gewesen.

Was die klinischen symptome der Krankheit anbelangt, so ist vor allem der schwere allgemeine Kräfteverfall zu betonen, der oft von starker psychischer Depression begleitet ist. Dies zeigt sich auch in Fällen von kürzerer Dauer und mit Ausgang in Genesung und muss als besonderes Charakteristicum der primären Angina gangraenosa gelten. Mackenzie (7) sagt, dass ihm diese Krankheit stets das Resultat einer Blutvergiftung $\mathrm{zu}$ sein scheine. Choleraartige Blässe, Kälte und Trockenheit der Haut und enorme Herzschwäche sind mehrfach beobachtet worden. Gubler (2) zählte bei seiner Patientin 24 Stunden vor dem Tode nur 18 Pulse in der Minute.

Die letal verlaufenden Fälle zeigen meist das Bild schwerer Sepsis und sind oft begleitet von schweren Gehirnerscheinungen: Benommenheit des Sensoriums, Delirien, soporöser Zustand, Diplopie [Trousseau (4)], stundenlanger Singultus bis zum Tode [Baudot (6)] finden sich in den Schilderungen der Autoren.

Der Foetor ex ore, eins der wichtigsten Symptome der Krankheit, ist nach Angabe der Autoren in seiner Stärke abhängig von der Ausbreitung der Rachengangrän. Sind nur kleine Bezirke befallen, so ist er gering oder kann ganz fehlen, bei diffuser Gangrän verpestet er zuweilen die ganze Umgebung und zwingt zur Isolirung des Kranken. Je später der Brandschorf sich abstösst, desto stärker wird seine putride Zersetzung und dementsprechend der Foetor.

Von Complikationen in den übrigen Körpertheilen wäre folgendes $\mathrm{zu}$ erwähnen. Gubler (2) beschreibt starke venöse Stauung in den Bauchorganen, ferner ein grosses Fibringerinnsel, das den rechten Ventrikel total ausfüllte, der Wand des Ventrikels fast unlösbar fest anhaftete und bis in das rechte Herzohr und die Vena cava superior hineinreichte. Intra vitam war ein leises Geräusch am Herzen gehört worden.

Mackenzie (7) beobachtete dreimal kurz vor dem Tode entstehendes Glottisödem, auch die Tracheotomie konnte nur vorübergehenden Nutzen schaffen. Von allen übrigen Autoren wird ein derartiges Ereigniss nicht erwähnt.

Bei Trousseau's Kranken entstanden in der vorletzten Woche Gliederschmerzen, die erst für rheumatische gehalten wurden. Nach 48 Stunden erkannte man, dass sie voll der Entzündung der oberflächlichen Venen hervorgerufen waren.

Bemerkenswerther ist das Vorkommen von Blutungen. Hierbei ist $\mathrm{zu}$ unterscheiden zwischen Blutungen aus Kapillaren und kleinen Venen der Hautoberfläche, sowie der serösen Häute und solcher aus grösseren Gefässen in Folge von Arrosion ihrer Wandungen durch die brandige Ulzeration. Letzere können wohl ge- legentlich so profus werden, dass sie unmittelbar den Tod herbeiführen.')

Blutungen aus einer vom Brand angefressenen Arteria pharyngea ascendens oder tonsillaris sind grob mechanisch erklärbar. Die kapillären Blutungen hingegen bedürfen der Voraussetzung einer durch toxische Einflüsse entstandenen Läsion der Kapillarwände oder bakterieller Embolieen und einer verminderten Gerinnungsfähigkeit des Blutes, an die schon Musset (5) gedacht $\mathrm{zu}$ haben scheint. Es werden beschrieben Hämorrhagieen in den Bulbus oculi und Hautpurpura [Luzet (9)]. Bei Musset entstand eine breite, die ganze reclite Thoraxhälfte bis in die Achselhöhle einnehmende schwärzlich marmorirte Ekchymose; sie ging später unter der inneren Darreichung von ferr. sesquichloratum zurück, als die lokale Rachenaffektion ebenfalls geheilt war. Blumenau (12) beschreibt punktförmige Blutungen in den serösen Häuten, im Endokard und in den weichen Hirnhäuten.

Ueber Verlauf und Dauer der Krankheit noch einige Bemerkungen! Sehr wichtig ist die Kenntniss der Thatsache, dass die Krankheit zuweilen in einzelnen Schüben auftritt, indem die erste Attacke anscheinend ausheilt, dann die Affektion wiederkehrt, sich vielleicht wiederum bessert, um dann nach einigen Tagen, Wochen oder Monaten von neuem aufzuloderll und nunmehr ihren wahren und malignen Charakter zu offenbaren. Hierbei kann die Rachenaffektion von vornherein gangränös sein oder anfänglich ganz harmlos und wie eine gewöhnliche katarrhalische oder follikuläre Angina aussehen, sodass man sich über den Ernst der Situation leicht längere Zeit Täuschungen hingeben kann. Ein derartiger Fall war derjenige von Musset (5), wo sechs Tage lang eine Angina mit rahmigem, breiartigem Belag auf beiden Mandeln bestand, die sich gebessert hatte, als plötzlich unter hohem Fieber und Delirien die Gangrän der Mandeln entstand.

Lastra (10) berichtet über einen Fall, wo die Krankheit $1 \frac{1}{2}$ Jahr dauerte, bis der Tod eintrat. Dieser Patient, ein 38jähriger Mann, hatte bereits fünf Monate lang an stechenden Schmerzen in der linken Mandel gelitten, als die Gangrän - schwärzlichgrau verfärbte Tonsille mit Kothgestank - festgestellt wurde. Durch lokale Applikation von Karbol und allgemeine Therapie wurde Besserung erzielt. Nach einem Jahre aber sah Lastra den Patienten mit ausgesprochenem Marasmus wieder. Die Gangrän hatte sich auf die benachbarten Rachentheile ausgedehnt und der Patient ging in kurzer Zeit zu Grunde.

Diese chronisch verlaufenden Fälle sind aber in der Minderheit. Gewöhnlich schwankt die Dauer der Krankheit, soweit Angaben vorliegen, zwischen zwei bis drei Wochen.

Ueber die Ausdehnung des brandigen Prozesses und ihren Einfluss auf die Prognose, giebt Maurin (8) an, dass von fünf Fällen circumskripter Gangrän vier genasen und einer starb, während in fünf anderen, wo sich der Brand auf mehr oder minder grosse Partieen des Rachens ausgedehnt hatte, zwei gesundeten und drei zu Grunde gingen. Darnach scheint die diffuse Rachengangrän eine besonders ernste Prognose zu geben. Allerdings tritt dieses Verhältniss in der nach Maurin entstandenen Kasuistik, soweit genügende anatomische Beschreibung gegeben wird, weniger deutlich zu Tage.

Die Diagnose ergiebt sich meist unschwer aus dem Vorhandensein des lokalen Brandes. Nur im Beginn, bevor die Gangrän sich zeigt, kann gelegentlich eine Verwechselung mit einer einfachen, nicht brandigen Angina vorkommen. Ferner kann, wie

1) Eine solche tödtliche Blutung aus einer Mandel bei einem 45 jährigen Neger beschreibt Cragin (New York med. Journal 1. September 1888). Hier hatte es sich aber offenbar nicht um primäre Angina gangraenosa gehandelt, sondern um phlegmonöse Tonsillitis. Diese hatte zu tiefen Ulzerationen und zur Arrosion zweier kleiner Blutgefässe geführt, deren Oeffnungen bei der Obduktion gefunden wurden. Intra vitam war es nicht gelungen, einen Ueberblick über den Rachen zu gewinnen, weil der Patient nicht im Stande war, den Mund weit genug zu öffnen. Bei der plötzlichen Blutung wurde aber zuerst blutiger Eiter entleert, was gegen primäre Gangrän spricht. Das Primäre scheint die eitrige Abszedirung der Mandel gewesen zu sein, sodass der Fall streng genommen, nicht hierher gehört.

Einen Fall von Rachenbrand im Gefolge einer phlegmonösen Tonsillitis erwähnt noch $\mathrm{K}$ unze (Lehrbuch der praktischen Medizin 1878, Bd. I, S. 251). Hier wurde nach fünf- bis sechswöchiger Dauer Heilung erzielt. Vergleiche auch Cozzolino (ref. intern. Centralblatt für Laryngologie 1885, Bd. I, S. 386.) 
schon erwähnt; die sekundäre Form des Rachenbrandes bei Tonsillitis phlegmonosa fälschlicher Weise für primäre Gangrän gehalten werden. Die starke Gewebsinfiltration und schliessliche Vereiterung sichern hier die Diagnose.

Schwieriger erscheint unter Umständen die Abgrenzung der Krankheit gegenüber einer in den letzten Jahren in Frankreich vielfach beschriebenen Rachenaffektion, der Angina Vincenti. ${ }^{1}$ ) Von diesem Autor wurde sie zuerst als „Angine diphthéroïde à bacilles fusiformes" beschrieben, von späteren Autoren als eine Form der Stomatitis ulcerosa membranacea bezeichnet, mit auf die Mandeln beschränkter Lokalisation. Die Kennzeichen dieser Krankheit sind eine grauliche Pseudomembran auf geschwürigem Grunde und in dieser massenhafte spindelförmige Bazillen meist in Verbindung mit Spirillen. Der Athem hat fötiden Geruch, der aber mit dem brandigen Fötor nicht verwechselt werden kann. Dieses sowie die Benignität - kein einziger Todesfall wurde beobachtet -, das nur wenig gestörte Allgemeinbefinden und der charakteristische bakteriologische Befund unterscheiden die Angina Vincenti von der primären Rachengangrän. ${ }^{2}$ )

Die Differentialdiagnose gegenüber der Diphtherie pflegt leicht zu sein. Im Gegensatz zu dieser zeigt die Gangrän keine Löffler'schen Bazillen, keine erhabenen, von der intakten Mandeloberfläche abziehbaren Membranen; vielmehr sind die Plaques bei der Gangrän nicht erhaben, sitzen fest in der Mandelsubstanz selbst und hinterlassen tiefe, zerklüftete Ulzerationen. Der aashafte Fötor besteht nur bei Gangrän. Endlich ist bei der Diphtherie die Schwellung der Halsdrüsen meist beträchtlich, besonders bei den Fällen mit ausgedehnter Membranbildung, während bei der Gangrän trotz der Schwere der Rachenaffektion diese Drüsenschwellung oft nur geringfügig bleibt.

Die Syphilis kann in allen ihren drei Perioden Erscheinungen auf den Gaumenmandeln machen. Besonders der syphilitische Primäraffekt einer Tonsille kann grosse Aehnlichkeit mit primärer Gangrän haben. Hierbei entsteht ein Geschwür mit schleimig eitrigem Belag unter sehr erheblichen Schluckschmerzen, sogar ein sekundärer Brand kann dazutreten. Auch schwere Allgemeinerscheinungen, Schüttelfrost, Fieber bis $\mathrm{zu} 40^{\circ}$, allgemeine Abgeschlagenheit und Kopfschmerz können vorkommen ${ }^{3}$ ) und die Aehnlichkeit vervollkommnen. Das wichtigste Kriterium ist, dass bei der Syphilis wenige Tage nach den ersten Beschwerden bereits massige, wenig empfindliche Drüsentumoren am Halse von Taubenei- bis Faustgrösse entstehen, Ferner stehen beim Schanker die Mandelsklerose und der Halsbubo oft in so inniger Verbindung, dass man, die Mandel und Halsdrüsen zwischen zwei Finger nehmend, den Eindruck eines zusammenhängenden Tumors bekommt. ${ }^{3}$ ) Immerhin kann die Differentialdiagnose vorübergehend fast unmöglich sein. In einem Falle von Maurin (8) ist diese Verwechselung vorgekommen.

Die Therapie ist in den schweren Fällen gänzlich machtlos. Für den Beginn ein Brechmittel [Bouchut (3)], Gurgelung mit den üblichen antiseptischen Mischungen, lokale Applikation von Glycerin (Bouchut), Aetzungen mit der bei den älteren französischen Autoren beliebten rauchenden Salzsäure, mit Argentum nitricum, Chromsäure etc. werden empfohlen. Innerlich sind Roborantien, insbesondere die Chinarinde, beliebte Medikationen. Musset (5) will, wie oben erwähnt, in seinem von der grossen Hautblutung betroffenen Falle durch innerliche Darreichung von Ferr. sesquichloratum in Dosen von $2 \mathrm{~g}$ pro die während dreier Tage Heilung erzielt haben. -

(Schluss folgt.)

1) Genaue Schilderung der Krankheit und Litteratur bei Nichlot et Marotte, Revue de Médecine 1901, S. 327. Vergl. auch Salom on, diese Wochenschrift 1899, No. 19 und 1901, No. 34.

2) Wie schwer diese Unterscheidung gelegentlich sein kann, lehrt der interessante Fall von Krebs (Deutsche medizinische Wochenschrift 1902, No. 17). Hier waren die Geschwüre auf der betroffenen Mandel sehr tief gegangen und es entstanden missfarbige nekrotische Fetzen, sodass ein Bild zu Stande $\mathrm{kam}$, das anatomisch wohl nicht von dem der Angina gangraenosa maligna zu unterscheiden war. Wie der Verfasser anerkennt, spricht aber das dauernd gute Allgemeinbefinden der Patientin bei dem zweimonatigen Verlauf sowie die prompte Heilung nach Entfernung der Mandel durchaus gegen primäre Gangrän. Ansserdem scheint der bakteriologische Befund für Angina Vincenti zu sprechen.

3) Vgl. Lieven, Die Syphilis der Mund- und Rachenhöhle, S. 42 u. 43 , in "Klinische Vorträge über Otologie etc.", herausg. von Haug. Bd. IV. 2. 